\title{
Antagonism of Central Growth Hormone-Releasing Factor Activity Selectively Attenuates Dark-Onset Feeding in Rats
}

\author{
Franco J. Vaccarino,' David Feifel, ${ }^{1}$ Jean Rivier, ${ }^{2}$ and Wylie Vale ${ }^{2}$ \\ 'Departments of Psychology and Psychiatry, University of Toronto, Toronto, Ontario, Canada M5S $1 \mathrm{~A} 1$ and ${ }^{2} \mathrm{Clayton}$ \\ Foundation Laboratories for Peptide Biology, Salk Institute for Biological Studies, La Jolla, California 92138
}

\begin{abstract}
Exogenous growth hormone-releasing factor (GRF) has been shown to activate feeding by central mechanisms involving the suprachiasmatic nucleus/medial preoptic area (SCN/ MPOA). Until now, however, the role played by endogenous GRF in the SCN/MPOA in naturally occurring eating has remained unknown. To investigate this, the effects of SCN/ MPOA injections of GRF antiserum (AS-GRF) on feeding in rats were studied. In light of SCN involvement in the control of circadian rhythms, AS-GRF was tested during the light and dark phases of the photoperiod. Results indicated that AS-GRF significantly attenuated dark-onset feeding but had no effect on feeding during the mid-dark, mid-light, or lightonset periods. These results suggest that endogenous GRF does play a role in naturally occurring feeding and that it may be involved in the circadian organization of feeding.
\end{abstract}

Animal studies investigating the behavioral effects of growth hormone-releasing factor (GRF) have shown that GRF plays a stimulatory role in feeding behavior (Vaccarino et al., 1985, 1988; Riviere and Bueno, 1986; Vaccarino and Buckenham, 1987; Vaccarino and Hayward, 1988; Feifel and Vaccarino, 1989; Dickson and Vaccarino, 1990). Intracerebroventricular administration of GRF, in doses that do not stimulate growth hormone $(\mathrm{GH})$ release, increases food intake without affecting other behaviors (Vaccarino et al., 1985, 1988; Feifel and Vaccarino, 1989). Peripheral administration of similar doses of GRF and central administration of a structurally related but inactive GRF analog have no effect on feeding (Vaccarino et al., 1985; Feifel and Vaccarino, 1989). Thus, the stimulatory effects of GRF on feeding behavior are centrally mediated and neurochemically and behaviorally specific.

In an effort to identify the central site of action mediating GRF-induced feeding, mapping studies have examined the effects of GRF injections aimed at a variety of hypothalamic sites known to contain GRF terminals. The results indicate that the suprachiasmatic nucleus/medial preoptic area (SCN/MPOA) region of the hypothalamus is a highly sensitive site for GRFinduced feeding (Vaccarino and Hayward, 1988; Dickson and Vaccarino, 1990). The fact that GRF exerts its effects at sites not directly associated with the portal blood system is consistent with anatomical (Sawchenko et al., 1985) and electrophysio-

\footnotetext{
Received May 3, 1991; revised July 24, 1991; accepted July 25, 1991.

This research was supported by NIH Grant DK26741 and NSERC Grant 35036.

Correspondence should be addressed to Franco J. Vaccarino, Department of Psychology, University of Toronto, 100 St. George Street, Toronto, Ontario M5S 1Al, Canada.

Copyright (c) 1991 Society for Neuroscience $0270-6474 / 91 / 113924-04 \$ 05.00 / 0$
}

logical (Twery and Moss, 1985) evidence that, in addition to its classic endocrine role in the pituitary, GRF has neurotransmitter-like properties in the brain. The importance of the SCN/ MPOA for mechanisms controlling circadian rhythms (Rusak and Zucker, 1979) combined with the fact that GRF-induced feeding is photoperiod sensitive (Feifel and Vaccarino, 1989) further suggests that GRF may be acting on mechanisms underlying the circadian regulation of feeding behavior. More specifically, we have hypothesized that elevated activity of cndogenous GRF in the SCN/MPOA may contribute to the increased feeding observed during the rat's active dark photophase (Feifel and Vaccarino, 1989).

Although the above studies show that exogenous GRF in the SCN/MPOA can influence feeding behavior, the extent to which endogenous GRF in the SCN/MPOA contributes to normal feeding behavior is not known. In order to assess the involvement of endogenous GRF in ongoing baseline feeding, the present study examined the effects of interference with endogenous GRF actions on feeding behavior. Because no specific and/or high-affinity antagonist for GRF exists, antiserum raised against GRF (AS-GRF) was utilized as a functional antagonist. Also, in light of the evidence that GRF's feeding effects are photoperiod sensitive and involve circadian mechanisms (Vaccarino and Hayward, 1988; Feifel and Vaccarino, 1988), the effects of intra-SCN/MPOA AS-GRF injections on food intake at four different time points across the light/dark cycle were examined.

\section{Materials and Methods}

Twenty-eight male Wistar rats (250-300 gm at surgery) were randomly assigned to one of two housing rooms. The rooms were in close proximity to each other, and both were illuminated according to a $12 \mathrm{hr}$ light $/ 12 \mathrm{hr}$ dark photoschedule. The rooms were identical except that their photoschedules were $180^{\circ}$ out of phase so that one room $(A)$ received lighting from 0700 to 1900 , while the other room (B) received lighting from 1900 to 0700 . Animals in each room were housed in individual cages and had free access to food (Purina rat chow) and water at all times throughout this study. Sixteen rats were housed in room $\mathrm{A}$, and 12 rats were housed in room $B$.

Each animal was surgically implanted with a unilateral, $23 \mathrm{ga}$, stainless steel guide cannula aimed $3 \mathrm{~mm}$ dorsal to the $\mathrm{SCN}$ region of the hypothalamus. Surgerics were conducted stercotaxically under Somnotal anesthesia $(65 \mathrm{mg} / \mathrm{kg}$ ) and utilized the following coordinates: $1.6 \mathrm{~mm}$ anterior, $2.2 \mathrm{~mm}$ lateral, and $7.2 \mathrm{~mm}$ ventral to bregma/skull surface, with the nose bars at $+5.0 \mathrm{~mm}$ above the intraaural line (Pellegrino et al., 1979). Cannulas were made to penetrate the brain medially at an angle of $15^{\circ}$ so as to avoid penetration of midline structures (Vaccarino and Hayward, 1988). Following surgery, subjects were returned to their home cages and allowed 3 weeks in which to recover and fully entrain to their respective photoschedules. During this period, animals were handled regularly.

In order to screen for GRF-induced feeding, animals were given in- 


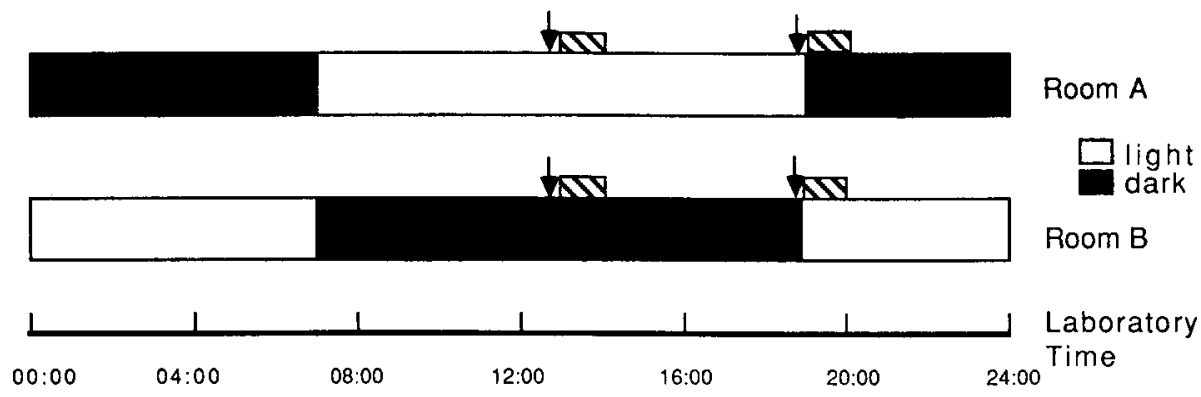

Figure 1. Experimental design. Arrows represent injections of AS-GRF and NRS into the SCN/MPOA. Hatched boxes represent $\mathrm{I} \mathrm{hr}$ measurement of food intake.

tra-SCN/MPOA injections of 0 (vehicle) and 4 pmol of rat hypothalamic rhGRF, following their postsurgical recovery period. Injections were administered in the middle of the light photoperiod. All injections were administered in a $0.25 \mu \mathrm{l}$ vehicle solution $(0.01 \%$ ascorbic acid in saline) via a 30 ga stainless steel injector cannula that protruded $3 \mathrm{~mm}$ ventral to the base tip of the guide cannula. Drug delivery was accomplished by a motorized microdrive system that injected $0.25 \mu$ lover a $60 \mathrm{sec}$ period. Following delivery, microinjectors were left in place an additional $30 \mathrm{sec}$. The order of injections followed a counterbalanced design with 1 drug-free $d$ between injections. Immediately following each injection, animals were returned to their home cages where food intake for the following $1 \mathrm{hr}$ was recorded. Food intake was measured by giving animals premeasured amounts of food and subtracting the weight of the amount of food remaining (plus spillage) at the end of the $1 \mathrm{hr}$ test.

Animals that consumed a minimum of $50 \%$ more food following GRF injections relative to vehicle injections were categorized as "responders"; the remaining subjects were categorized as "nonresponders."

Following another drug-free day, testing with rabbit antiserum raised against GRF (AS-GRF) and normal rabbit serum (NRS) began. Animals housed in room $A$ were tested at the mid-light and dark-onset time points. Animals housed in room B were tested at mid-dark and lightonset time points. All animals were tested with both NRS and AS-GRF in a counterbalanced order, at their respective time points, with a minimum of 2 drug-free $d$ between injections (see Fig. 1). Beginning $20 \mathrm{~min}$ after injections, $1 \mathrm{hr}$ of food intake was measured. In this way, food intake measurements were obtained for four different time points throughout the photocycle: light onset, mid-light, dark onset, and middark.

AS-GRF was produced in rabbits using rat hypothalamic GRF coupled to human $\alpha$-globulins with bisdiazotized benzidine as immunogen, absorbed with human $\alpha$-globulins, and lyophilized. Both AS-GRF and NRS were administered as $1 \%$ solutions (diluted in saline) in a $0.25 \mu \mathrm{l}$ volume. This volume and dilution were arrived at from pilot testing of various dilutions that revealed that solutions of higher concentration had a viscosity that significantly hindered microinjections. Furthermore, the small volume has been found in our experience to result in limited drug spread to neighboring sites (Vaccarino and Hayward, 1988; Dickson and Vaccarino, 1990). Including the GRF trials, subjects received a total of six central injections.

After completion of the final feeding test, the effects of AS-GRF on general locomotor activity at dark onset were assesed. Subjects in room $B$ were given an additional set (AS-GRF and NRS) of two injections (AS-GRF and NRS) at dark onset, $4 \mathrm{~d}$ after the final fecding trial. Injections were carried out in counterbalanced order with a minimum of 2 drug-free $d$ between injections. Following each injection, rats were placed in locomotor aclivity cages equipped with dual photobeam-sensing systems that measured the number of photobeam interruptions for $1 \mathrm{hr}$. Food and water were unavailable during this time.

At the conclusion of the study, animals were perfused with saline and then $10 \%$ formalin ( $5 \%$ sucrose). Brains were removed, sectioned 40 $\mu \mathrm{m}$ thick, and stained with cresyl violet to determine cannula placements. The analysis was carried out by an investigator without knowledge of which sections belonged to animals in the responder and nonresponder groups.

The collected data were submitted to statistical analysis consisting of individual Student's $t$ test comparisons between NRS and AS-GRF treatments at each of the four photoperiod times. The statistical critcrion for significance was $p<0.05$.

\section{Results}

The amount of food consumed following intra-SCN/MPOA injections of rhGRF administered in the middle of the light photoperiod for subjects classified as responders and nonresponders is represented in Figure 2.

Figure 3 illustrates the cannula placcments for each of the subjects. Placements for all of the responders (13/13) were within the SCN/MPOA region. In contrast, the nonresponders were characterized by placements outside the SCN/MPOA. Thirteen of the 15 nonresponders had placements outside the SCN/MPOA. Two nonresponders had placements that fell on the outer borders of the MPOA. In general, tissue damage surrounding the cannula tip was minimal-never greater than a $0.3 \mathrm{~mm}$ distance from the microinjector tip.

Baseline food intake did not differ significantly between responder and nonresponder groups at any of the four times tested: $0.30 / 0.27,0.72 / 0.79,2.70 / 3.01$, and $1.91 / 1.81 \mathrm{gm}$ for responders/nonresponders during the early-light, mid-light, early-dark, and mid-dark test periods, respectively.

Figure 4 illustrates food intake following intra-SCN/MPOA injections of NRS and AS-GRF during the four times tested. Dark-onset food intake (measurcd during the first hour of the dark cycle) was significantly reduced $[t(6)=3.75 ; p<0.01]$ following SCN/MPOA injections of AS-GRF in comparison to normal serum. At none of the other three photoperiod times tested were there any significant effects of AS-GRF on the amount of food consumed.

No significant effects of AS-GRF on food intake were noted

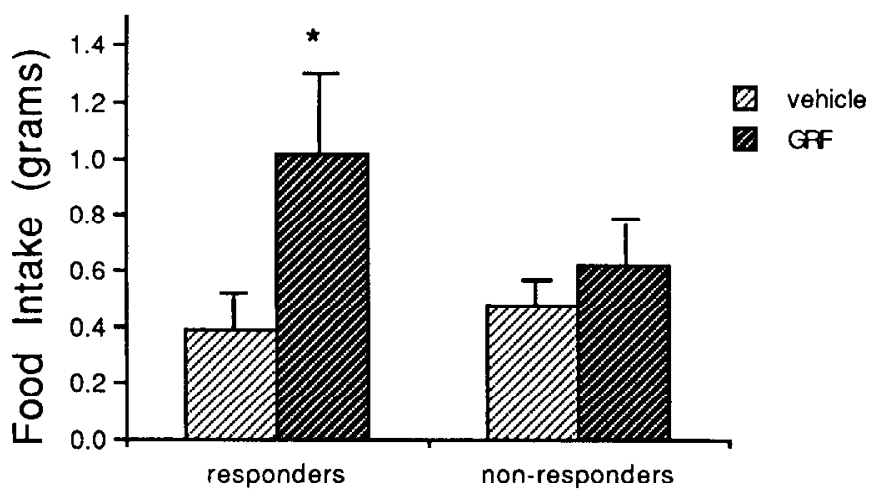

Figure 2. The effects of intra-SCN/MPOA administration of GRF on food intake during the middle of the light cycle. Bars represent the mean ( \pm SEM) amount of food eaten by subjects who were subsequently classified as responders $(n=13)$ and nonresponders $(n=15)$, during the hour following intra-SCN/MPOA injections of vehicle or GRF (4 P.M.). *, Significantly $(p<0.01)$ different from vehicle treatment. 
Figure 3. Microinjection sites, based on histological examination, for animals classified as responders (solid squares) and nonresponders (open squares). Numbers represent anterior level from bregma (in $\mathrm{mm}$ ). $A C$, Anterior commissure; $D B B$, diagonal band of Broca; $L V$, lateral ventricle; $M P O A$, medial preoptic area; $O C$, optic chiasm; $P O A$, preoptic area; $S C N$, suprachiasmatic nucleus; $3 r d V$, third ventricle.
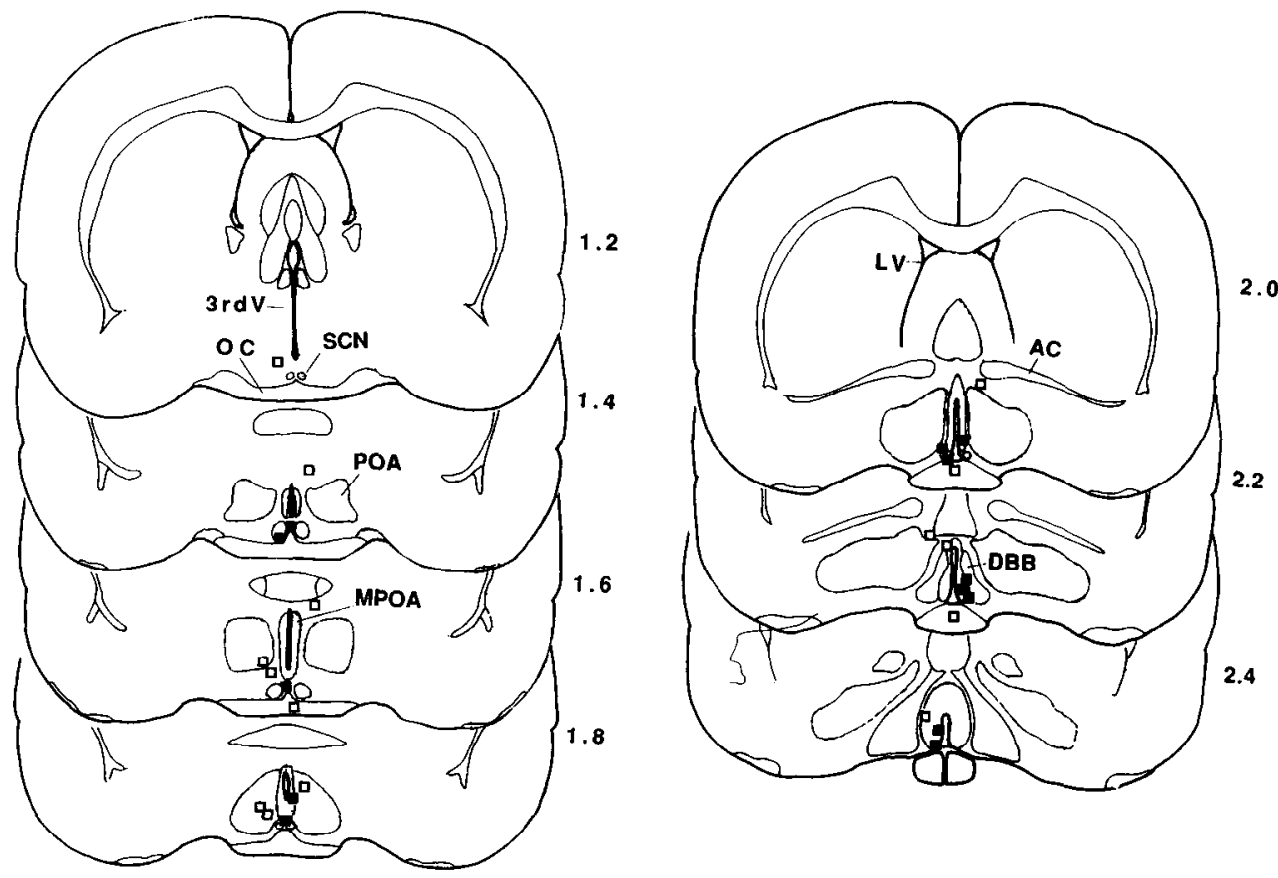

at any of the four photocycle times tested in subjects classified as nonresponders (Fig. 5).

Locomotor tests revealed that AS-GRF injections administered to responders just prior to dark onset resulted in general locomotor activity [22.5 \pm 6.7 (SEM) photobeam interruptions] that was not significantly different $(p=0.66)$ from that displayed following injections of NRS (16.5 \pm 7.8 photobeam interruptions).

\section{Discussion}

Rats display a circadian feeding pattern in which the vast majority of their daily food intake is consumed during the dark. Within this general, nocturnally dominant, circadian feeding pattern, rats display an ultradian pattern of intermittent meals.
The largest single feeding episodes occur at dark-onset and latedark parts of the photoperiod (Rossenwasser et al., 1979). Baseline feeding (i.e., following NRS treatments) seen during this study is consistent with this established circadian pattern.

The present results demonstrate that interference with $\mathrm{SCN} /$ MPOA GRF at the onset of the dark cycle attenuates the feeding burst associated with this part of the photocycle. The importance of the SCN/MPOA region in this effect is underlined by the fact that the feeding response to GRF (facilitation) or AS-GRF (inhibition) could predict the anatomical accuracy of the intraSCN/MPOA injection. The behavioral specificity of the inhibition of feeding observed following AS-GRF treatment at dark onset is evidenced by the lack of any AS-GRF effect on general locomotor activity during this time.

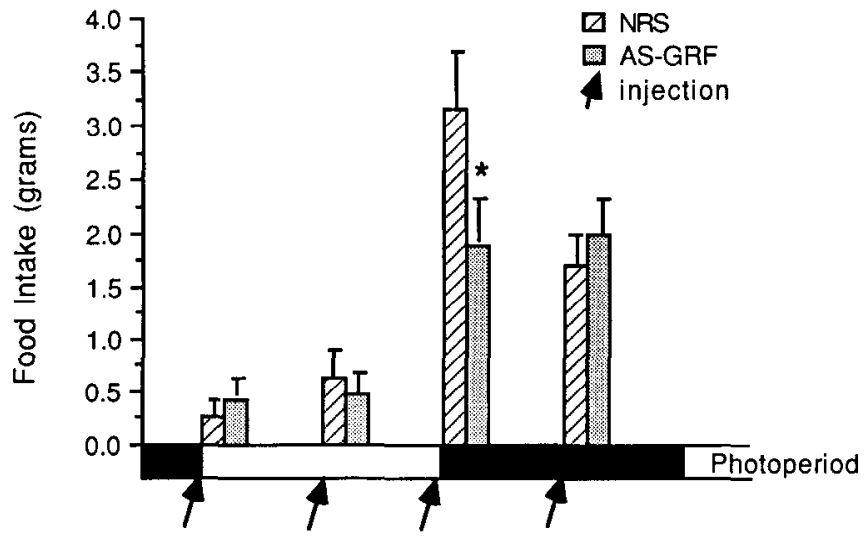

Figure 4. The effects of rabbit antiserum raised against rhGRF (AS GRF) and of NRS injected into the SCN/MPOA on food intake in responders. Bars represent mean $( \pm$ SEM) food intake for $\mathbf{l}$ hr during the light onset $(n=6)$, the mid-light $(n=7)$, the dark onset $(n=7)$, and mid-dark $(n=6)$, following injection of AS-GRF or NRS into the SCN/MPOA. *, Significantly different $(p<0.01)$ from corresponding vehicle treatment.

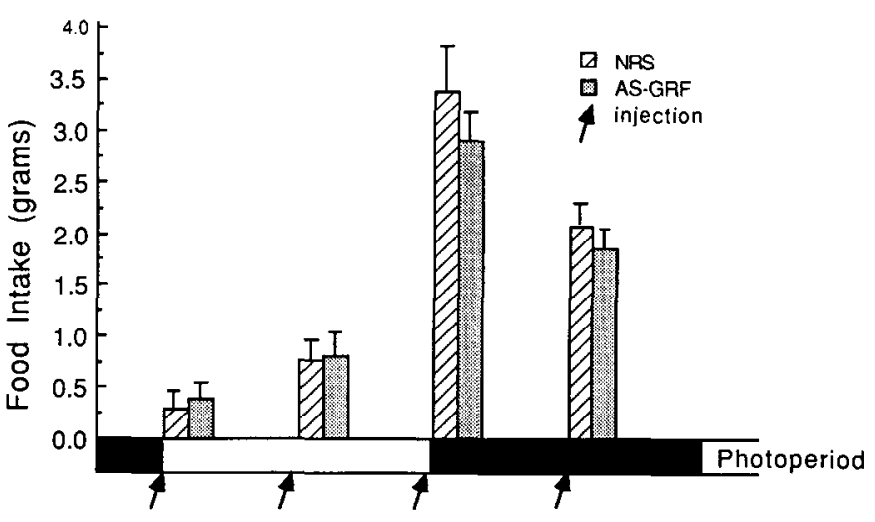

Figure 5. The effects of rabbit antiserum raised against rhGRF (ASGRF) or of NRS injected into the SCN/MPOA on food intake in animals classified as nonresponders. Bars represent mean ( \pm SEM) food intake for $1 \mathrm{hr}$ during the light onset $(n=6)$, mid-light $(n=9)$, dark onset $(n$ $=9$ ), and mid-dark $(n=6)$, following injection of AS-GRF or NRS into the SCN/MPOA. 
Combined with the fact that the SCN/MPOA is a sensitive site for GRF-induced feeding (Vaccarino and Hayward, 1988; Dickson and Vaccarino, 1990; present results), the present results support the notion that increased endogenous GRF activity in the SCN/MPOA is, at least partly, responsible for the elevated food intake observed in free-feeding rats at the onset of the active (dark) phase of the photocycle (Feifel and Vaccarino, 1989). However, the inability of AS-GRF to affect feeding when applied in the middle of the dark cycle suggests that the neural factors triggering dark-phase feeding are not identical to those maintaining it and that GRF plays a significant role in the former but not the latter.

Dark-onset feeding following AS-GRF, though significantly attenuated, did not revert to the low levels seen during the less active, light photophase. It remained comparable to mid-dark levels. This suggests that GRF does not contribute to the increased feeding seen throughout the dark and that GRF plays a role in the hyperphagic peak seen at the initiation of the dark photoperiod. This is consistent with the notion that increased GRF activity in the SCN/MPOA during the onset of dark may act as a signal triggering nocturnal feeding mechanisms (Feifel and Vaccarino, 1989). Alternatively, a separate GRF feeding mechanism may acutely act in an additive fashion with pandark feeding mechanisms.

The lack of any significant change in feeding following ASGRF injections during the light suggests that, though feeding is sensitive to the effects of exogenous GRF in the light (Vaccarino et al., 1985, 1988; Vaccarino and Hayward, 1988; Feifel and Vaccarino, 1989; Dickson and Vaccarino, 1990), endogenous GRF does not play a significant role in the low level of feeding displayed during in this photoperiod. This is also consistent with the theory that endogenous GRF contributes to the increased feeding observed during the dark and that exogenous GRF potentiates feeding in the light by acutely activating these nocturnal feeding mechanisms (Feifel and Vaccarino, 1989).

An alternate explanation, however, is also plausible. It is possible that GRF's involvement in feeding is coupled with the ultradian pattern of $\mathrm{GH}$ release. This is supported by findings indicating that some (but not all) feeding episodes are coupled with GH peaks (Even et al., 1987). It may be, therefore, that the mid-dark point chosen in our study did not represent an optimal time point relative to $\mathrm{GH}$ peaks. Thus, testing of additional time points during the dark (or the light for that matter) might have revealed GRF involvement in spontaneous feeding at other time points.

GH secretion in the rat and many other species is expressed as a ultradian pattern of pulses (Tannenbaum and Martin, 1976). These pulses have been shown to be entrained to the light/dark cycle since a $\mathrm{GH}$ pulse is expressed consistently around the onset of the dark photophase (Even et al., 1987). The fact that the ultradian meal pattern in rats is also entrained to the light/dark cycle, with the most active peak in eating also occurring around dark onset, is indirect evidence that the mechanisms governing $\mathrm{GH}$ secretion and feeding behavior are linked. Studies that have shown a strong temporal correspondence between meals and GH secretion provide further evidence for this (Uvnas-Moberg et al., 1984; Even et al., 1987). The finding that the SCN/MPOA, a brain region critical to the circadian organization and photoentrainment of both $\mathrm{GH}$ secretion (Willoughby and Martin,
1978) and meals (Rusak and Zucker, 1979), is a highly sensitive site for GRF-induced feeding suggests that GRF is a likely candidate to underlie the coordination of feeding and GH release.

The central appetitive effects, together with evidence for central effects on gut motility (Bueno et al., 1985), further suggest that GRF may underlie a broad integration of central and peripheral aspects of metabolic/energy regulation and somatic growth. By manipulating endogenous GRF levels, this study provides direct evidence that endogenous GRF does indeed play a role in the central regulation of naturally occurring feeding. The temporal specificity of the AS-GRF-induced blockade of feeding strengthens previous evidence (Vaccarino and Hayward, 1988; Feifel and Vaccarino, 1989) that endogenous GRF contributes, in part at least, to the circadian organization of feeding and acts as a trigger shifting feeding from the low levels seen during the light to the higher levels seen during the dark.

\section{References}

Bueno L, Fioramonti J, Primi MP (1985) Central effects of growth hormone-releasing factor (GRF) on intestinal motility in dogs: involvement of dopaminergic receptors. Peptides 6:403-407.

Dickson PR, Vaccarino FJ (1990) Characterization of feeding behavior induced by central injection of GRF. Am J Physiol 259:R651-R657.

Even PJ, Danguir S, Nicolaidis C, Rougeot C, Dray F (1987) Pulsatile secretion of growth hormone and insulin in relation to feeding in rats. Am J Physiol 253:R772-R778.

Feifel D, Vaccarino FJ (1989) Feeding effects of growth hormonereleasing factor in rats are photoperiod sensitive. Behav Neurosci 103: 824-830.

Feifel D, Vaccarino FJ (1990) Central somatostatin: a re-examination of its effects on feeding. Brain Res 535:189-194.

Pellegrino LJ, Pellegrino AS, Cushman AS (1979) A stereotaxic atlas of the rat brain. New York: Plenum.

Riviere P, Bueno L (1986) Influence of regimen and insulinemia on orexigenic effects of GRF(1-44) in sheep. Physiol Behav 39:347-350.

Rossenwasser AM, Boulous Z, Terman M (1979) Circadian organization of food intake and meal patterns in the rat. Physiol Behav 27: 33-39.

Rusak B, Zucker I (1979) Neural regulation of circadian rhythms. Physiol Rev 59:449-526.

Sawchenko PE, Swanson LW, Rivier J, Vale WW (1985) The distribution of growth hormone-releasing factor immunoreactivity in the central nervous system of the rat: an immunohistochemical study using antisera directed against rat hypothalamic GRF. J Neurol 237: $100-115$.

Tannenbaum GS, Martin JB (1976) Evidence for an endogenous ultradian rhythm governing growth hormone secretion. Endocrinology 99:720-727.

Twery MJ, Moss RL (1985) Sensitivity of rat forebrain neurons to growth hormone-releasing hormone. Peptides 6:609-613.

Uvnas-Moberg K, Heidvall K, Grenback E, Hulting A (1984) Release of GH in response to feeding in dogs. Acta Physiol Scand 121:8991.

Vaccarino FJ, Buckenham KE (1987) Naloxone blockade of growth hormone-releasing factor-induced feeding. Regul Pept 18:165-171.

Vaccarino FJ, Hayward M (1988) Microinjections of growth hormone-releasing factor into the medial preoptic area/suprachiasmatic nucleus region of the hypothalamus stimulate food intake in rats. Regul Pept 21:21-28.

Vaccarino FJ, Bloom FE, Rivier J, Vale W, Koob GF (1985) Stimulation of food intake in rats by centrally administered hypothalamic growth hormone-releasing factor. Nature 314:167-168.

Vaccarino FJ, Feifel D, Rivier J, Vale W, Koob GB (1988) Centrally administered growth hormone-releasing factor stimulates food intake in free-feeding rats. Peptides [Suppl 1] 9:35-38.

Willoughby JO, Martin JB (1978) The suprachiasmatic nucleus synchronizes growth hormone secretory rhythms with the light-dark cycle. Brain Res 151:413-417. 